\title{
An Accurate Moment Method Model for the Tapered Slot Antenna
}

\author{
RAMAKRISHNA JANASWAMY, MEMBER, IEEE
}

\begin{abstract}
A moment method model is presented for the radiation characteristics of the tapered slot antenna. It overcomes the shortcoming of an earlier theory. The method is rigorous for the air dielectric antennas. For dielectric supported antennas, the method is approximate, but accurate. The method is particularly accurate for antennas etched on substrates that are electrically thin or those having a low dielectric constant, within the limits of $\left(\epsilon_{r}-1\right) d / \lambda_{0} \leq 0.1$. Numerical results are presented for the radiation pattern and compared with experimental results to validate the model.
\end{abstract}

\section{INTRODUCTION}

$\mathrm{A}$ N APPROXIMATE ANALYSIS of the tapered slot antenna shown in Fig. 1(a) has been presented in [1]. The antenna consists of a thin film of metal (hereafter shown as hatched areas) with or without a dielectric substrate on one side of the film. A major drawback of the previous theory is that the model is valid only for large values of the antenna height $2 H^{\prime}$, typically requiring it to be at least six free-space wavelengths. However, it was demonstrated experimentally in [1] that narrower main beams could be obtained by constructing antennas with smaller heights. In this paper, we present a moment method (MM) model for the tapered slot antenna. The model is valid for arbitrary antenna heights. The present model is rigorous for antennas built without a dielectric substrate. For antennas on a dielectric substrate, the model is approximate, but nevertheless accurate. The presence of the dielectric is included in the MM solution via the impedance boundary conditions [2].

The present model is based on the MM solution of the electric field integral equation for the electric surface currents flowing on the conducting plates of the antenna. This is in contrast to the aperture field (electric field within the tapered slot) modeling of [1]. In order to properly model the surface currents of the antenna of Fig. 1(a), quadrilateral or triangular patches must be used to segment the plates. This will result in a large number of unknowns in the MM solution. In order to keep the unknowns to a manageable number and to simplify the analysis, a slightly different geometry of the antenna is considered as shown Fig. 1(b). This geometry facilitates the use of the simpler rectangular patches in the MM solution, and will be adopted throughout this paper. This, however, does not change the physics that governs the radiation mechanism of

Manuscript received July 20, 1988; revised January 3, 1989. This work was supported by the Naval Postgraduate School Foundation Research Program.

The author is with the Department of Electrical and Computer Engineering, Naval Postgraduate School, Code 62JS, Monterey, CA 93943.

IEEE Log Number 8929312.

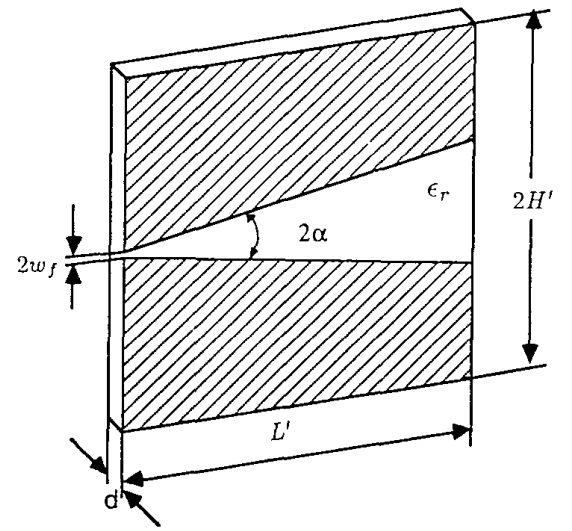

(a)

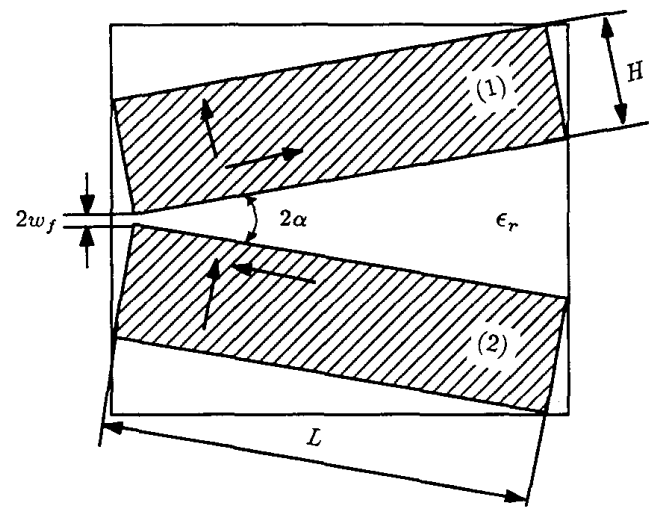

(b)

Fig. 1. Geometry of the tapered slot antenna. (a) Original problem. (b) Modified structure treated here.

the antenna. In Section II, we present a brief theory describing the model. In Section III, numerical results are presented and comparison is made with experiment to validate the model.

\section{Moment Method Model}

Fig. 1(b) shows the geometry of the problem. $L$ and $H$ denote the length and height of the antenna, respectively. The substrate has a relatively permittivity $\epsilon_{r}$ and has a thickness $d$. The antenna is assumed to be in the transmitting mode with an impressed source (voltage or current) at the feed gap. The starting point in the analysis is the reaction integral equation for the surface current [3]. An $e^{j \omega t}$ time dependence is assumed for all fields and sources and is suppressed throughout. 
Consider the impressed sources $\left(\vec{J}_{i}, \vec{M}_{i}\right)$ bounded by a volume $V$ and radiating in the presence of the antenna surface $S$. The reaction integral equation states that [3]

$$
\begin{aligned}
& \iint_{S}\left(\vec{J}_{S} \cdot \vec{E}_{T}-\vec{M}_{s} \cdot \vec{H}_{T}\right) d s \\
&=-\iiint_{V}\left(\vec{J}_{i} \cdot \vec{E}_{T}-\vec{M}_{i} \cdot \vec{H}_{T}\right) d v
\end{aligned}
$$

where $\left(\vec{E}_{T}, \vec{H}_{T}\right)$ is the free space field of an electric test source $\vec{J}_{T}$ placed on $S$, and $\left(\vec{J}_{s}, \vec{M}_{s}\right)$ represent the total surface currents on the antenna. For conductors of finite conductivity, the impedance boundary condition

$$
\vec{M}_{s}=Z_{s} \vec{J}_{s} \times \hat{n}
$$

is used, where $Z_{s}$ is the surface impedance [4]. $\vec{M}_{s}$ vanishes if the antenna surface is a perfect conductor. Equation (1) states that there is a zero reaction between a test source $\vec{J}_{T}$ on the surface and the currents $\left(\vec{J}_{s}, \vec{M}_{s}\right)$ and $\left(\vec{J}_{i}, \vec{M}_{i}\right)$ which generate the total field.

The integral equation (1) is solved by the method of moments for the unknowns $\left(\vec{J}_{s}, \vec{M}_{s}\right)$. Detailed steps leading to the development of the model are described below.

\section{A. Antenna Without a Dielectric Substrate}

This case is termed the air dielectric case. In this case the plates comprising the antenna are assumed to be perfect conductors. Thus $\vec{M}_{s} \equiv \overrightarrow{0}$ and only electric surface current flows on the conducting plates. The unknown $\vec{J}_{s}$ is expanded in a set of $N$ basis functions (expansion functions) of the form

$$
\vec{J}_{s}=\sum_{n=1}^{N} I_{n} \vec{J}_{n}
$$

and (1) is enforced for $N$ test sources residing on $S$. This procedure discretizes (1) to a set of simultaneous linear equations for the unknown coefficients $I_{n}$

$$
\sum_{n=1}^{N} I_{n} Z_{m n}=V_{m}, \quad m=1,2, \cdots, N
$$

where

$$
Z_{m n}=-\iint_{n} \vec{J}_{n} \cdot \vec{E}_{m} d s
$$

and represents the mutual impedance between the $m$ th testing mode and the $n$th expansion mode, and

$$
V_{m}=\iiint_{V}\left(\vec{J}_{i} \cdot \vec{E}_{m}-\vec{M}_{i} \cdot \vec{H}_{m}\right) d v .
$$

The rectangular plates 1 and 2 are subdivided into rectangular patches [5] and overlapping surface dipoles are used to represent the surface currents along the two principal directions of each plate as shown in Fig. 2(a). The current on each surface dipole is assumed to be piecewise sinusoidal (PWS) in the direction of current flow and constant transverse to it

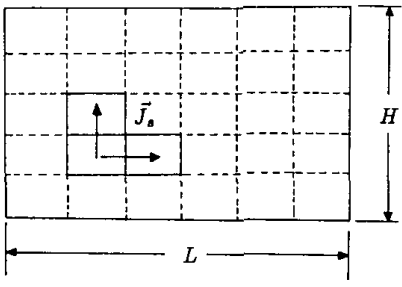

(a)

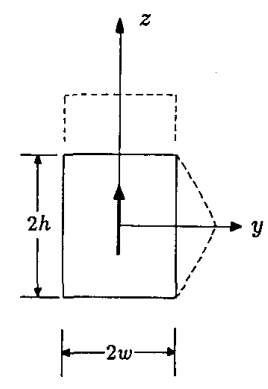

(b)
Fig. 2. (a) Plate segmentation. (b) PWS surface dipole.

[6], that is,

$$
\vec{J}_{s}=\hat{z} \frac{1}{2 w} \frac{\sin k_{0}(h-|z|)}{\sin k_{0} h}, \quad|y|<w,|z|<h
$$

where $k_{0}=2 \pi / \lambda_{0}$, and $\lambda_{0}$ is the free space wavelength. Fig. 2(b) shows a pictorial representation of the expansion modes. The test modes are chosen identical to the expansion modes, and thus, the method is a Galerkin's method resulting in a symmetric impedance matrix.

The surface current on each plate has two components: a component that flows along the length $L$ of the antenna, which we term as the transmission line mode $J_{\text {TRA }}$, and a component that flows along the height $H$, which we term as the radiating mode $J_{\mathrm{RAD}}$. Owing to the symmetry of the antenna structure about a horizontal plane, the net vertical currents are of equal magnitudes and have the same directions on the two plates, whereas the net horizontal currents are of equal amplitudes, but have opposite directions on the plates. Thus, the directions of $J_{\mathrm{TRA}}$ and $J_{\mathrm{RAD}}$ are as indicated in Fig. 1(b). If $N_{L}$ and $N_{H}$ denote the number of segments on each plate along the length and the height, respectively, the total number of modes on each plate is $N_{H}\left(N_{L}-1\right)+N_{L}\left(N_{H}-1\right)$.

The main computational task in the MM modeling is the computation of the impedance matrix elements of (3). The mutual impedance between parallel surface dipoles is found using the expression provided in [6]. When the two coplanar surface dipoles are nonparallel as shown in Fig. 3, we adopt the procedure outlined in [5]. The surface dipoles are assumed to be comprised of filamentary dipoles located arbitrarily on each surface patch. Mutual impedance between the two surface dipoles is then found by integrating across each patch the mutual impedance between the filamentary dipoles. The mutual impedance between two arbitrarily oriented coplanar PWS filamentary dipoles is given in [7]. For the current distribution assumed in (5), the mutual impedance between the surface dipole- 1 and the surface dipole- 2 that are inclined at a mutual angle of $\psi$ is obtained as

$$
\begin{gathered}
Z_{12}=\frac{-3.75}{\sin k_{0} h_{1} \sin k_{0} h_{2}} \sum_{k=1}^{3} \sum_{l=1}^{3} \sum_{p=-1,2}^{2} \sum_{q=-1,2}^{2} p q C_{k} D_{l} \\
\cdot \int_{-1}^{1} \int_{-1}^{1} E\left[k_{0}\left(R_{k l}+p z_{k}+q r_{l}\right)\right] e^{j k_{0}\left(p z_{k}+q r_{l}\right)} d u d v .
\end{gathered}
$$

The various terms involved are $C_{1}=C_{3}=D_{1}=D_{3}=$ 


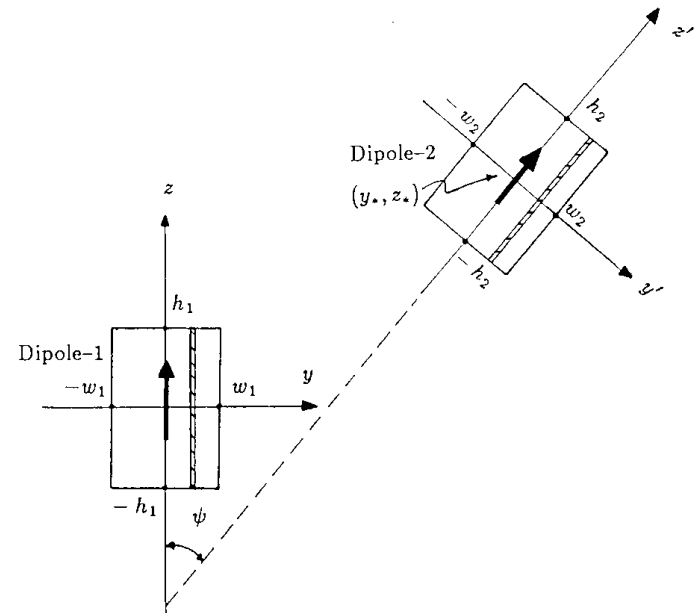

Fig. 3. Geometry for calculating the mutual impedance between nonparallel surface dipoles.

$1, C_{2}=-2 \cos k_{0} h_{1}$, and $D_{2}=-2 \cos k_{0} h_{2}$. The lengths of dipole- 1 and dipole- 2 are $2 h_{1}$ and $2 h_{2}$, respectively. Further,

$$
\begin{gathered}
R_{k l}=\left(r_{l}^{2}+z_{k}^{2}-2 r_{l} z_{k} \cos \psi\right)^{1 / 2} \\
r_{l}=r_{1}+(l-2) h_{2} \\
r_{1}=-u w_{1} \csc \psi+v w_{2} \cot \psi+y_{*} \csc \psi \\
z_{k}=z_{1}+(k-2) h_{1} \\
z_{1}=-u w_{1} \cot \psi+v w_{2} \csc \psi+y_{*} \cot \psi-z_{*} .
\end{gathered}
$$

$E(\cdot)=\mathrm{Ci}(|\cdot|)-j \mathrm{Si}(\cdot)$ is the exponential integral, and $2 w_{1}$ and $2 w_{2}$ are the respective widths of dipole-1 and dipole- 2 . $\left(y_{*}, z_{*}\right)$ are the coordinates of the center of surface dipole-2 in the coordinate system of surface dipole-1 (see Fig. 3).

Two different source models are considered. In the first model, an infinitesimal current element is placed at the center of the feed gap as shown in Fig. 4(a). The current element does not make contact with the surface of the antenna structure and no conditions are imposed regarding the continuity of the current at the feed gap. The current element induces surface currents on the antenna surface, and the scattered field together with the field due to the current element constitutes the radiation pattern of the antenna.

In a second model, which is useful in computing the input impedance of the antenna as well, a thin strip dipole, with a delta gap generator at its center, is superimposed on the antenna structure at the feed gap (see Fig. 4(b)). The length and the width of the strip dipole are $2 L_{d}$ and $2 w_{d}$, respectively. The length of the strip dipole is chosen long enough so as to connect the two plates of the antenna and provide a continuity of the current from one plate to the other.

It is felt that the second source model is more useful, as it enables the calculation of the input impedance of the antenna. This model introduces an additional unknown in the form of the strip dipole current. The influence of the strip dipole on the radiation pattern of the antenna is expected to be significant only in the backlobe region, where the surface currents on

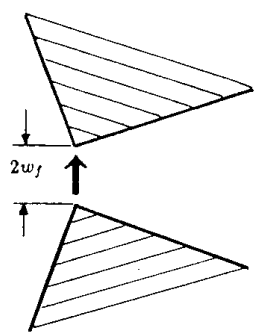

(a)

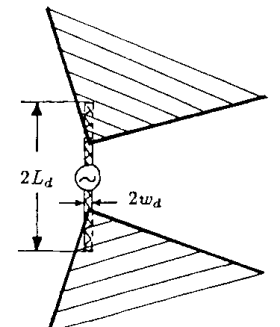

(b)
Fig. 4. Source modeling. (a) Infinitesimal current source at the feed gap. (b) Delta-gap generator with a strip dipole at the feed gap.

the plates contribute little. In the results to follow, the delta gap source model will be assumed. A direct connection (i.e., without the strip dipole) of the delta gap generator across the feed gap of the antenna was not considered. The use of a delta gap generator presupposes that the shape of the structure at the contact point is free of corners. The antenna under consideration has a right angled corner at the feed gap. To circumvent this problem, the delta gap generator was imposed via the strip dipole.

\section{B. Antenna on a Dielectric Substrate}

Experience suggests that the presence of a dielectric substrate has the primary effect of narrowing the main beam of the antenna [1]. The dielectric slows down the currents on the plates, as is typical in an endfire-producing surface wave antenna. A MM model that includes the dielectric rigorously in the analysis is complicated and greatly increases the number of unknowns. We shall, however, adopt an approximate model based on the use of impedance boundary conditions on a shell [2]. We treat the presence of the dielectric in the following two steps.

1) Step 1: The dielectric coated plates comprising the antenna are no longer treated as perfectly conducting, but as having a surface impedance $Z_{s}$. This surface impedance relates the tangential electric field to the surface currents on a finitely conducting surface. For a dielectric coated plane conductor, it follows from [2] that $Z_{s}=j\left(\eta_{0} / 2 \sqrt{\epsilon_{r}}\right) \tan k_{0} d \sqrt{\epsilon_{r}}$, where $\eta_{0} \simeq 120 \pi$ is the intrinsic impedance of free space. This value of $Z_{s}$ is taken in (2) and in the integral equation (1). In the MM solution of (1), this would result in an impedance matrix of the form $[Z]+[\Delta Z]$ where $[Z]$ is the impedance matrix of section $A$ (i.e., without the dielectric substrate). A typical term of $[\Delta Z]$ would be

$$
\Delta Z_{m n}=Z_{s} \iint \vec{J}_{n} \cdot \bar{J}_{m} d s .
$$

We see that $\Delta Z_{m n}$ is zero unless the expansion and testing modes are parallel and overlapping. For the assumed current distribution of (5), closed-form expressions can be obtained for $\Delta Z_{m n}$. The result is

$$
\begin{aligned}
& \Delta Z_{m n}=Z_{s} \frac{h}{4 w \sin ^{2} k_{0} h} \\
& \begin{cases}2\left(1-\frac{\sin 2 k_{0} h}{2 k_{0} h}\right), & m=n \\
\left(\frac{\sin k_{0} h}{k_{0} h}-\cos k_{0} h\right), & |m-n|=1 .\end{cases}
\end{aligned}
$$


2) Step 2: The presence of the dielectric further results in volume polarization current $\vec{J}_{p}=j \omega \epsilon_{0}\left(\epsilon_{r}-1\right) \vec{E}$ in the tapered slot region (and other "uncovered" regions) of the antenna.This polarization current contributes to the radiated field along with the surface currents induced on the plates. Numerical experiments have revealed that the contribution of $J_{p}$ is only a second-order effect unless the substrate is electrically thick or has a high permittivity. This was experimentally confirmed by comparing the radiation pattern of the antenna by retaining the dielectric with that obtained by removing the dielectric form the tapered slot region. The study has indicated that the effect of polarization current is negligible for $\left(\epsilon_{r}-1\right) d / \lambda_{0} \leq 0.04$ (approximately). The quantity $\vec{E}$ in the expression for $\vec{J}_{p}$ is the total electric field. However, due to the reasons mentioned above, $\vec{E}$ is taken as the field that is obtained by using Step 1 alone. Radiation due to the polarization current is then calculated by integrating $\vec{J}_{p}$ over the tapered slot region with the far-field free space Green's function as the kernel. Hence, as far as the MM solution is concerned, only Step 1 affects the determination of the surface current distribution.

\section{Numerical Results and Discussion}

A computer program was developed that computes the current distribution and the radiation pattern of the antenna for given values of $L, H, w_{f}, \epsilon_{r}, d, L_{d}, w_{d}$, and at a specified frequency $f$. Symmetry of the structure and Toeplitz symmetry resulting from the Galerkin's method were incorporated in the code. The validity of the code was checked by favorably comparing to the literature the results for the radar cross section of a rectangular conducting plate.

It was found that four to five segments per wavelength along either principal directions of the rectangular plate were sufficient to obtain convergent results for the radiation pattern. A larger number of segments is needed for the convergence of the current distribution. Other characteristics such as the input impedance may be directly obtained from the current distribution. The CPU time spent on a VAX 11/785 machine to compute each impedance matrix element was around $2 \mathrm{~s}$.

Fig. 5 shows the radiation patterns of an air dielectric tapered slot antenna having $L=17.3 \mathrm{~cm}, H=3 \mathrm{~cm}, w_{f}=1$ $\mathrm{mm}$, and $\alpha=7^{\circ}$. The frequency of operation was $f=9$ GHz. At this frequency, $L \approx 5.2 \lambda_{0}$ and $H=0.9 \lambda_{0}$. The computed pattern (dot-dashed curve) is compared with the experimentally measured pattern (solid line). The experimental model was built using a 5 mil brass sheet, and the antenna was supported on a 1-in styrofoam sheet $\left(\epsilon_{r} \simeq 1.04\right)$. The antenna was used in a receiving mode. A microwave detector diode (HP-2082-2794) was soldered at the feed gap to pick the 1 $\mathrm{kHz}$ modulated RF signal. In the MM model, the strip dipole parameters were chosen as $L_{d}=3.33 \mathrm{~mm}$ and $w_{d}=0.333$ $\mathrm{mm}$. At the given frequency of $9 \mathrm{GHz}$, the overall length of the strip dipole is $0.2 \lambda_{0}$. The number of segments were $N_{L}=21$ and $N_{H}=4$, resulting in 143 modes on each plate. It is seen from Fig. 5 that there is good agreement between the two. The slight discrepancy away from the main lobe region is attributed to radiation from the strip dipole at the feed. Numerical studies have revealed that this discrepancy decreases

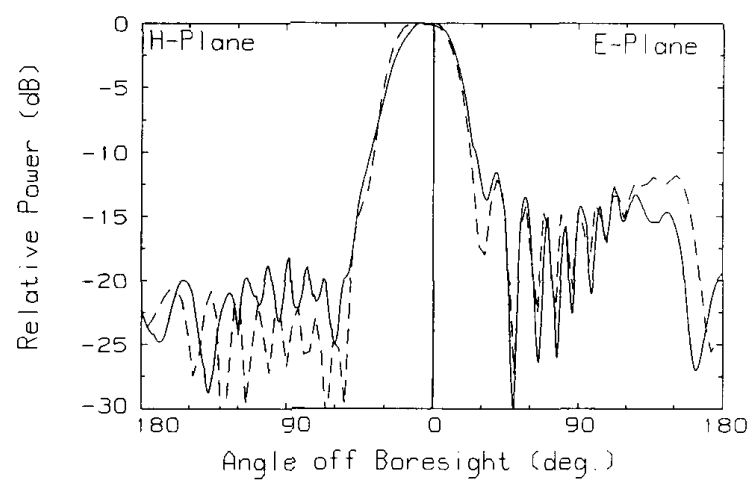

Fig. 5. Comparison of radiation pattern between the theory and experiment for an air dielectric tapered slot antenna. $L=5.2 \lambda_{0}, H=0.9 \lambda_{0}$, and $\alpha=7^{\circ}$. Solid line-measured, dot-dashed line-theory.

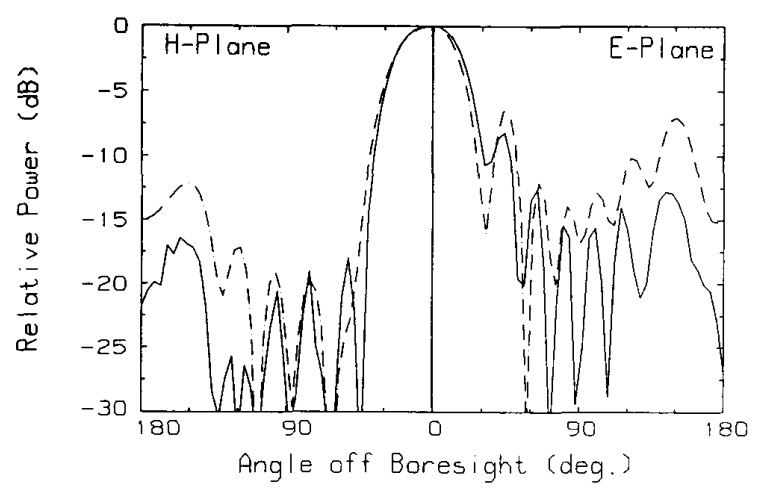

Fig. 6. Comparison of pattern for dielectric supported antenna. $L=$ $3 \lambda_{0}, H=0.9 \lambda_{0}, \alpha=6^{\circ}, \epsilon_{r}=2.33$ and $d=0.021 \lambda_{0}$. Solid line-measured, dot-dashed line-theory.

as the length of the feed dipole is decreased. A length at least $0.2 \lambda_{0}$ was chosen so as to avoid any numerical instabilities that may result during the impedance matrix computation (for instance, a short strip dipole would have a very large capacitive input impedance. Additionally, it gives rise to relatively small mutual impedances with other elements). The total CPU time needed on a VAX $11 / 785$ to obtain the solution for the current distribution was around $9 \mathrm{~h}$.

Fig. 6 shows the comparison between the theory (dotdashed curve) and experiment (solid curve) for an antenna etched on a 28 mil, $\epsilon_{r}=2.33, \mathrm{RT} /$ Duroid 5870 substrate. For this antenna $L=10 \mathrm{~cm}, H=3 \mathrm{~cm}, w_{f}=0.75 \mathrm{~mm}, \alpha=6^{\circ}$, and $f=9 \mathrm{GHz}$. At this frequency, $L=3 \lambda_{0}$ and $H=0.9$ $\lambda_{0}$. In the MM model, the strip dipole parameters were chosen as $L_{d}=3.33$ and $w_{d}=0.3 \mathrm{~mm}$. The number of plate segments were $N_{L}=15$ and $N_{H}=5$ resulting in 130 modes on each plate. Further, with the above substrate parameters, the surface impedance $Z_{s}=j 25.3 \mathrm{ohms}$. This inductive surface impedance correctly introduces a slow wave factor in the current distribution and narrows the main beam of the antenna. The experimental model was etched on a $10.25 \times 8.25$ $\mathrm{cm}$ board of copper clad substrate. It is seen from Fig. 6 that the computed radiation pattern agrees favorably with the measured pattern, except for some region in the backlobe. Further numerical studies have indicated that the discrepancy 
in the backlobe region is slightly sensitive to the length of the feed strip dipole. The currents on the plates radiate mainly in the end-fire direction, whereas the radiation due to the strip dipole is donut shaped. Its influence is, therefore, most significant where the main surface currents radiate little, viz., near the backlobe region. Since the strip dipole is only a theoretical artifice, we conclude that the discrepancy seen in the figure is due to radiation from the strip dipole. It is also worth mentioning that the contribution due to polarization was found to be insignificant in this case. Pattern calculated without this contribution produced an almost identical result as that shown in Fig. 6.

Fig. 7 shows the magnitude and phase of the radiating and transmission currents along two principal cuts on the top plate. All parameters are the same as those in Fig. 6. A unit volt delta-gap generator is assumed as the source. In Fig. 7(a), the cut passes through the center and is parallel to the length $L$ of the plate (cut AA). The current is mostly traveling wave in nature as is evident from the figure. The phase cross-over points are slightly nonequidistant. This is due to the relatively few basis functions (five per wavelength) used in the computation. An increase in the number of basis functions increases enormously the computational task involved, but without much improvement in the results for the radiation pattern.

Fig. 7(b) shows the variation of the currents along the other principal cut (cut $\mathrm{BB}$ ). It is seen from the phase distribution that, on the cut $\mathrm{BB}$, the current is primarily standingwave in nature. It is also interesting to see that the transmission mode current decays away from the $\zeta=0$ edge of the plate. The transmission mode current is largely confined to a small region of the plate in the vicinity of the tapered slot. Such a feature was also noticed for other antenna parameters.

Comparisons have also been made for antennas etched on 25 mil, $\epsilon_{r}=6$ and 10 mil $\epsilon_{r}=10.5$ substrates. The frequency of operation was $9 \mathrm{GHz}$ and the other antenna parameters remained the same as in the antenna of Fig. 6. Fig. 6 is indicative of the kind of agreement obtained with these antennas. Comparison was also made with the experimental data reported in [8]. In [8], the antenna was etched on a $2 \times 1$ in board of 25 mil thick Alumina substrate $\left(\epsilon_{r}=9.8\right)$, and its geometry was the same as in Fig. 1(a). Other parameters of the antenna were $f=9 \mathrm{GHz}, L \approx 3.5 \mathrm{~cm}, \alpha=5.7^{\circ}$, and $w_{f}=0.127 \mathrm{~mm}$. In order to make a comparison with the present model (that is valid for Fig. 1(b)), $H$ was assumed to be one half of the board height, viz., $1.25 \mathrm{~cm}$. It was found that whereas a very good agreement was obtained for the $E$-plane pattern, a rather poor agreement was obtained in the $H$-plane. In particular, the computed radiation pattern correctly predicted an $E$-plane 3 $\mathrm{dB}$ beamwidth of $40^{\circ}$ and a sidelobe level of $-10 \mathrm{~dB}$. In the $H$-plane, the computed radiation pattern predicted a $3 \mathrm{~dB}$ beamwidth of $97^{\circ}$ and a sidelobe level of $-5 \mathrm{~dB}$. Experimental data of [8] suggest corresponding values of $50^{\circ}$ and -10 $\mathrm{dB}$ for these quantities. Unsuccessful attempts were made to duplicate the experimental results of [8]. It is felt that the discrepancy is probably due to the way in which the dielectric substrate is treated in the present model. The dielectric will have to be modeled rigorously before definitive conclusions can be drawn about the disagreement in the $H$-plane pattern.

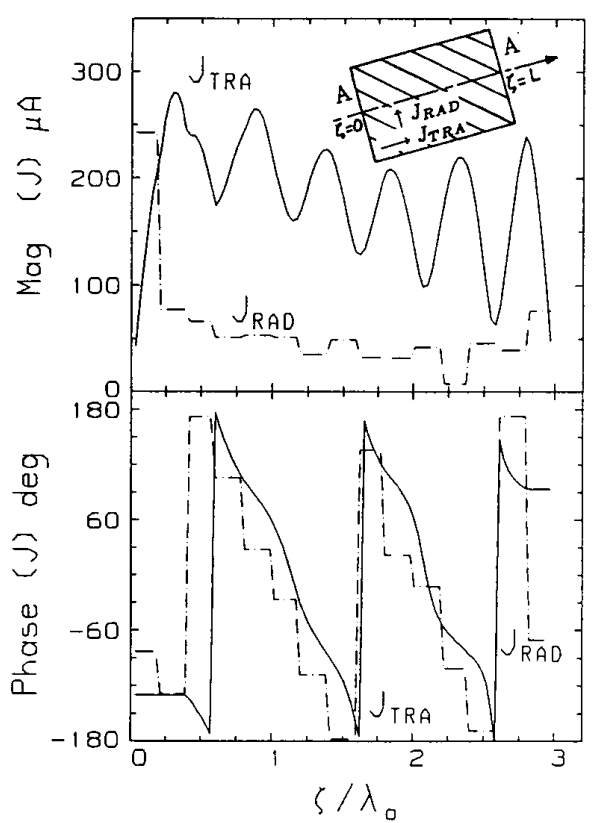

(a)

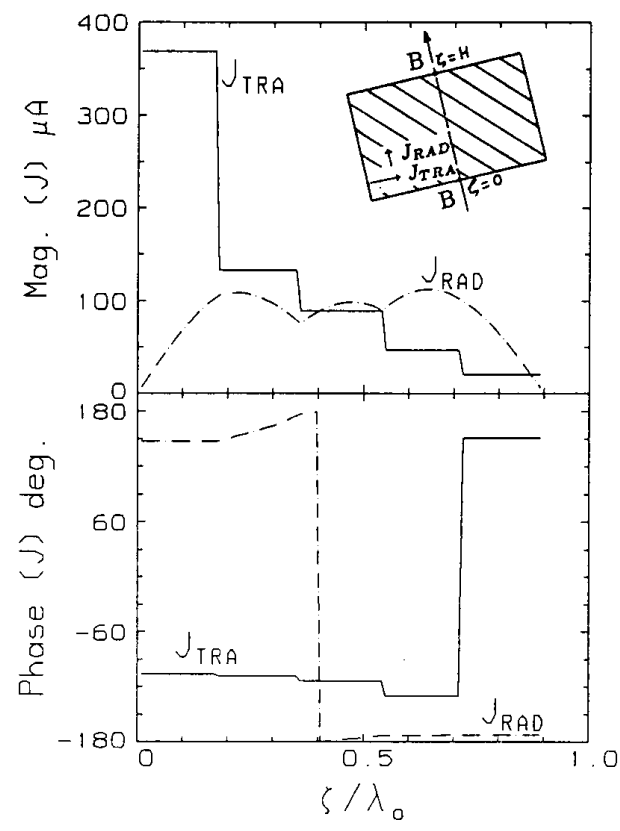

(b)

Fig. 7. Amplitude and phase variations of the current distribution on the principal cuts. $L=3 \lambda_{0}, H=0.9 \lambda_{0}, \alpha=6^{\circ}, \epsilon_{r}=2.33, d=$ $0.021 \lambda_{0}, N_{L}=15$, and $N_{H}=5$. (a) Cut-AA. (b) Cut-BB.

Based on the above results, it is felt that the present method yields sufficiently accurate results for dielectric substrates approximately satisfying $\left(\epsilon_{r}-1\right) d / \lambda_{0} \leq 0.1$. The upper limit corresponds to $\epsilon_{r}=6$ and $d / \lambda_{0}=0.02$.

\section{CONCLUSION}

A moment method model has been presented for the radiation characteristics of the tapered slot antenna. The method 
is rigorous for the air dielectric antennas. For dielectric supported antennas, the method is approximate and is based on modifying the air dielectric case by the use of the impedance boundary conditions. Accurate results are obtained both for the air dielectric case and for the dielectric supported case. The method appears to yield accurate results for dielectric supported antennas within the approximate limits of $\left(\epsilon_{r}-\right.$ 1) $d / \lambda_{0} \leq 0.1$. Results have been presented for a linear taper. However, with an appropriate choice of surface patches such as triangular subdomains, the method could be used to treat other taper and structural geometries of the antenna. The principal limitation of the present method is that large CPU times are needed to obtain the solution. With the ever increasing growth of fast microprocessor chips, this should pose no problem in the future.

\section{ACKNOWLEDGMENT}

The author would like to thank Professor Daniel $\mathbf{H}$. Schaubert for providing the experimental results presented in this paper, and for many fruitful discussions that increased the quality of this paper. His involvement is gratefully acknowledged. The author would also like to thank Dr. R. S. Tomar of Bolriet Technologies, Inc., Ontario, Canada, for conducting experiments related to the present work.

\section{REFERENCES}

[1] R. Janaswamy and D. H. Schaubert, "Analysis of the tapered slot antenna," IEEE Trans. Antennas Propagat., vol. AP-35, no. 9, pp. 1058-1064, Sept. 1987.

[2] K. M. Mitzner, "Effective boundary conditions for reflection and transmission by an absorbing shell of arbitrary shape," IEEE Trans. Antennas Propagat., vol. AP-16, no. 6, pp. 706-712, Nov. 1968.

[3] N. N. Wang, J. H. Richmond, and M. C. Gilreath, "Sinusoidal reaction formulation for radiation and scattering from conducting surfaces," IEEE Trans. Antennas Propagat., vol. AP-23, no. 3, pp. 376-382, May 1975

[4] T. B. A. Senior, "Impedance boundary conditions for imperfectly conducting surfaces," Applied Sci. Res., sec. B, vol. 8, no. 5-6, pp. 418-436, 1960.

[5] E. H. Newman and D. M. Pozar, "Considerations for efficient wire/surface modeling," IEEE Trans. Antennas Propagat., vol. AP28 , no. 1, pp. 121-124, Jan. 1980.

[6] R. Janaswamy, "A simplified expression for the self/mutual impedance between coplanar and parallel surface monopoles," IEEE Trans. Antennas Propagat., vol. AP-35, no. 10, pp. 1174-1176, Oct. 1987.

[7] J. H. Richmond and N. H. Geary, "Mutual impedance between coplanar skew dipoles," IEEE Trans. Antennas Propagat., vol. AP-18, no. 3, pp. 414-416, May 1970.

[8] S. N. Prasad and S. Mahapatra, "A novel MIC slot-line antenna," presented at 9th Eur. Micro. Conf., Brighton, U.K., 1979, pp. 120-124.

Ramakrishna Janaswamy (M'88), for a photograph and biography please see page 1064 of the September 1987 issue of this Transactions. 